\title{
Orthography Influences the Perception of Speech in Alexic Patients
}

\author{
Kimberly M. Miller*,1,2 and Diane Swick ${ }^{2,3}$
}

\begin{abstract}
Interactive models of reading propose that phonological representations directly activate and/or constrain orthographic representations through feedback. These models also predict that spoken words should activate their orthographic forms. The effect of word orthography on auditory lexical access was investigated in two patients with alexia without agraphia. Several theories of alexia suggest that letter-by-letter reading results from impaired access to orthographic representations. Although alexics can often correctly identify orally spelled words and spell to dictation, it is unknown whether they can access the whole orthographic "word-form" as a unit via auditory presentation. The nonobligatory activation of orthography was examined in an auditory lexical decision task, in which the orthographic and
\end{abstract}

\section{INTRODUCTION}

Models of visual word recognition attempt to explain how orthographic, phonological, and semantic codes are activated and coordinated to support the reading of words. A major distinguishing feature of different models is the hypothetical direction and strength of activation between these three domains. In feedforward models, processing proceeds primarily in one direction: from stimulus feature-extraction to higher-level cognitive processes (reviewed in Jacobs \& Grainger, 1994). For example, Carr and Pollatsek's (1985) parallel coding systems model postulates three parallel systems that work independently of one another. There is one orthographic pathway mediated by whole-word meanings, one mediated by the parsing of the whole word into morphemes, and a third pathway based on phonological recoding. This model is illustrated by 9 boxes, 13 onedirectional arrows, and only 1 bidirectional arrow, indicating mutual interaction between the semantic memory system and the hypothesized phonological decision mechanism. Thus, information is flowing primarily in

\footnotetext{
${ }^{1}$ University of California, Berkeley, ${ }^{2}$ Veterans Affairs Northern California Health Care System, ${ }^{3}$ University of California, Davis *Current address: Department of Clinical and Health Psychology, University of Florida, Gainesville, FL.
}

phonological similarity between prime and target was manipulated. In controls, the combined effect of phonological and orthographic relatedness (OP) produced greater facilitation than phonological relatedness alone, indicating that orthography can influence auditory lexical decisions. The alexics displayed patterns of facilitation comparable to controls, suggesting they can quickly access whole-word orthographic information via the auditory modality. An alternate account posits that the OP advantage does not require on-line access of orthography, but instead is a developmental by-product of learning to read an orthographically inconsistent language. The results have implications for cognitive theories of alexia and provide support for interactive models of word recognition.

one direction, with no recurrent feedback between higher- and lower-level processes.

In contrast, interactive models hypothesize that output from a process can be fed back to an earlier processing level to affect later input. These models propose that not only do orthographic representations feed forward to activate phonological representations for word pronunciation, but that phonological representations directly activate and/or constrain orthographic representations through feedback (Ziegler \& Ferrand, 1998; Stone, Vanhoy, \& Van Orden, 1997; Plaut, McClelland, Seidenberg, \& Patterson, 1996; Coltheart, Curtis, Atkins, \& Haller, 1991; Seidenberg \& McClelland, 1989). Thus, these models postulate a bidirectional flow of activity.

Theories of auditory word recognition have been even less inclined to incorporate any connection from phonology to orthography. Some models of speech recognition are fully modular (e.g., Norris, McQueen, \& Cutler, 2000). Unless one is engaged in an overt spelling task, it is not intuitively obvious why a word's orthography would be activated. Nevertheless, interactive models of word recognition predict that spoken words should activate their orthographic representations (Ziegler \& Ferrand, 1998; Stone et al., 1997). In accord with these models, several lines of evidence favor an influence of orthography on auditory lexical access. For example, Jakimik, Cole, and Rudnicky (1985) used a prime-target paradigm in an auditory lexical decision task and 
manipulated the prime's orthographic and phonological relatedness to the target word. Reaction times (RTs) to target words were faster for targets that were both orthographically and phonologically related (OP) to their primes (e.g., "fantasy-fan") than to words that were unrelated to their primes (e.g., "blanket-pill"). Furthermore, this facilitation was not observed when the prime and the target were related by sound alone (e.g., "definite-deaf'), indicating an additive effect of orthography and phonology.

In other experiments (Donnenwerth-Nolan, Tanenhaus, \& Seidenberg, 1981; Seidenberg \& Tanenhaus, 1979), participants monitored lists of spoken words for a target that rhymed with a cue word or performed rhyme-nonrhyme decision tasks. The critical manipulation was whether the target word was orthographically similar or different from the cue word (e.g., "pie-tie" and "rye-tie," respectively). Monitoring latencies to detect orthographically different rhymes were longer than latencies to detect orthographically similar rhymes, whether the cue words were presented aurally or visually. Likewise, rhyming pairs were judged to be rhymes more rapidly when they were spelled alike than when they were spelled differently (Seidenberg \& Tanenhaus, 1979). Conversely, nonrhymes which were spelled alike took longer to be judged as nonrhymes than those which were not orthographically similar. Thus, the orthographic word-form was accessed in tasks that could be performed without orthographic information. The authors interpreted this as evidence of an automatic activation of orthography by auditory lexical input.

One limitation of this study is the possibility that using rhyme monitoring and detection tasks induced subjects to use strategies that rely upon orthographic information that normally would not be accessed. To control for this problem, Tanenhaus, Flanigan, and Seidenberg (1980) conducted an experiment using a Stroop-type paradigm. A spoken prime word could be phonologically related (PR), orthographically related (OR), phonologically and orthographically related (OP), or unrelated (UN) to the target word. The target word was then presented visually, and the task was to name the "color" the target word was printed in as quickly as possible. All of the related conditions interfered with color naming, but the OP condition produced the greatest amount of interference. The fact that subjects accessed orthographic and phonological codes, even though it had a negative effect on performance, was taken as evidence that these codes did not become available through a conscious strategy; rather, they were accessed automatically as a consequence of word recognition.

What can an acquired reading disorder tell us about access to orthographic codes? Alexia without agraphia (also known as pure alexia) is a phenomenon in which reading is impaired, but writing remains intact. A key characteristic of pure alexia is a word length effect: As additional letters are added to a word string, the word- naming latency increases proportionally (Miozzo \& Caramazza, 1998). This suggests that the capacity for parallel processing of letter arrays is disrupted in alexics, leaving them to rely on a laborious method of stringing individual letters together to spell out a word. Patients who exhibit this characteristic are referred to as letterby-letter (LBL) readers. A deficit that typically manifests after a lesion to the left occipital cortex, pure alexia was first classified as a "disconnection syndrome" by Dejerine (1892). According to this interpretation, a right visual field cut produced by damage to the left occipital cortex prevents any visual input to the left hemisphere. Additionally, visual information that enters through the right occipital lobe cannot be transferred to left hemisphere language areas due to damage to the splenium of the corpus callosum.

Not all current theories agree with Dejerine's initial hypothesis. The level(s) of processing that are impaired in alexics is still debated. The "peripheral view" hypothesizes that the deficit occurs early in the reading system, prior to the activation of an orthographic representation (Behrmann, Plaut, \& Nelson, 1998). In contrast, the "central view" claims that the deficit takes place at a stage after basic visual processing. However, even the "central" theorists have placed the impairment at different levels. Warrington and Shallice (1980) have argued that alexics are unable to read whole words due to damage to a left hemisphere visual word-form system, so they must resort to LBL reading. Patterson and Kay (1982) suggest that the visual word-form system itself is intact but disconnected from the letter-form analysis system. While both these models postulate impaired whole-word orthographic access in alexia, other models do not. Saffran and Coslett (1998) hypothesize that LBL reading is supported by portions of the left hemisphere that are still intact, but the right hemisphere mediates whole-word reading via a direct connection from orthography to semantics.

The question we pose in the current study is, if alexics are unable to access in parallel the letters in a word presented visually, can they access in parallel the orthographic information contained in a word presented aurally? As reviewed above, the covert activation of orthographic representations appears to play a role in auditory rhyme priming effects. Although the alexics have relatively intact orthographic knowledge that supports their ability to spell orally presented words, presumably, the activation of visually based, whole-word orthography from auditory input would be compromised. If this process plays a role in rhyme priming for spoken words, then the alexic patients would be impaired. Conversely, if the OP effect is based on abstract (not visually based) orthographic representations, then it may be intact in the patients.

To assess the importance of a well-functioning visual input lexicon to rhyme priming, the current experiment utilized a prime-target presentation and an auditory 
lexical decision task (adapted from: Baum \& Leonard, 1999; Leonard \& Baum, 1997). The objective was twofold: first, to replicate Leonard and Baum's (1997) findings that control participants exhibit greater priming when the target-prime pair was OP (as opposed to only PR), suggesting that orthography has a facilitatory effect on auditory lexical access; and second, to see whether our two alexic patients displayed this same pattern of priming. The key comparison was between the PR condition (e.g., "drawn-gone"), and the OP condition (e.g., "tell-bell"). The only difference between these two conditions is that orthographic similarity is present in the latter. If word orthography is indeed activated by phonology, but alexics have impaired access to this information, it is predicted that they would perform equally fast under these two conditions. That is, orthography would not be available to make a contribution beyond that of phonology alone. However, if the alexics have intact access to orthographic representations, they would show an advantage in the OP condition, similar to controls. The results are discussed in relation to theories of alexia and models of word recognition.

\section{RESULTS}

\section{Control RT Data}

Participants were instructed to make lexical decisions to the second stimulus of aurally presented prime-target pairs. Each pair consisted of a real word prime, followed by a target that could be either a nonword or a real word. Control RT data for real word targets (taken from correct responses only) were analyzed by using repeatedmeasures analyses of variance (ANOVAs) with one withingroup factor, Prime Type. This factor had four levels: PR (e.g., "cloud-crowd"), OR (e.g., "deaf-leaf'), OP (e.g., "barn-yarn"), and UN (e.g., "realm-bridge"). Extreme RT values (defined as less than or greater than 2 standard deviations from the average RT, calculated per subject, per condition) were excluded from the analyses. Separate ANOVAs with both subjects $\left(F_{1}\right)$ and items $\left(F_{2}\right)$ as random factors were performed, and Min $F^{\prime}$ values were calculated for reasons delineated in Clark (1973). Planned comparisons (contrasts) were used to further describe significant effects. Greenhouse-Geisser corrected $p$ values are reported for all analyses.

Figure 1 shows the mean RTs according to Prime Type. There was a significant main effect of Prime Type in all three analyses, $F_{1}(3,36)=18.30, p<.0001$; $F_{2}(3,72)=9.27, p<.0002 ; \operatorname{Min} F^{\prime}(3,108)=6.15, p<$ .0007. Planned comparisons for subjects revealed that RTs under OP were significantly faster than under each of the other word priming conditions (all $p$ 's $<.03$; for OP vs. UN, $p<.0001$ ). Additionally, RTs under PR were significantly faster than under the UN condition, $F(1,12)$ $=14.54, p<.002$. Of particular interest was the planned comparison between the OP condition and the PR condition. RTs under OP were significantly faster than under the PR condition, $F(1,12)=6.84, p<.03$, suggesting an additional facilitatory effect when orthography is combined with phonology.

\section{Comparison of Patients to Controls}

Within the individual patients, RT data were compared with control RT data in order to assess whether Patients E.A. and A.L. performed slower than controls in general (Figure 1). Collapsing across all real word priming conditions, Patient E.A. was comparable to controls (975 msec for E.A.; $994 \mathrm{msec}$ for controls, ns), but Patient A.L. (1173 msec) fell outside the upper 99\%
Figure 1. RT data for controls and patients under each priming condition. $\mathrm{PR}=$ phonologically related; $\mathrm{OR}=$ orthographically related; $\mathrm{OP}=$ orthographically and phonologically related; $\mathrm{UN}=$ unrelated; $\mathrm{PN}=$ phonologically related nonword; UNN = unrelated nonword. Two asterisks indicate that the patient is outside the $99 \%$ confidence interval for controls. The error bars indicate the 95\% confidence interval.

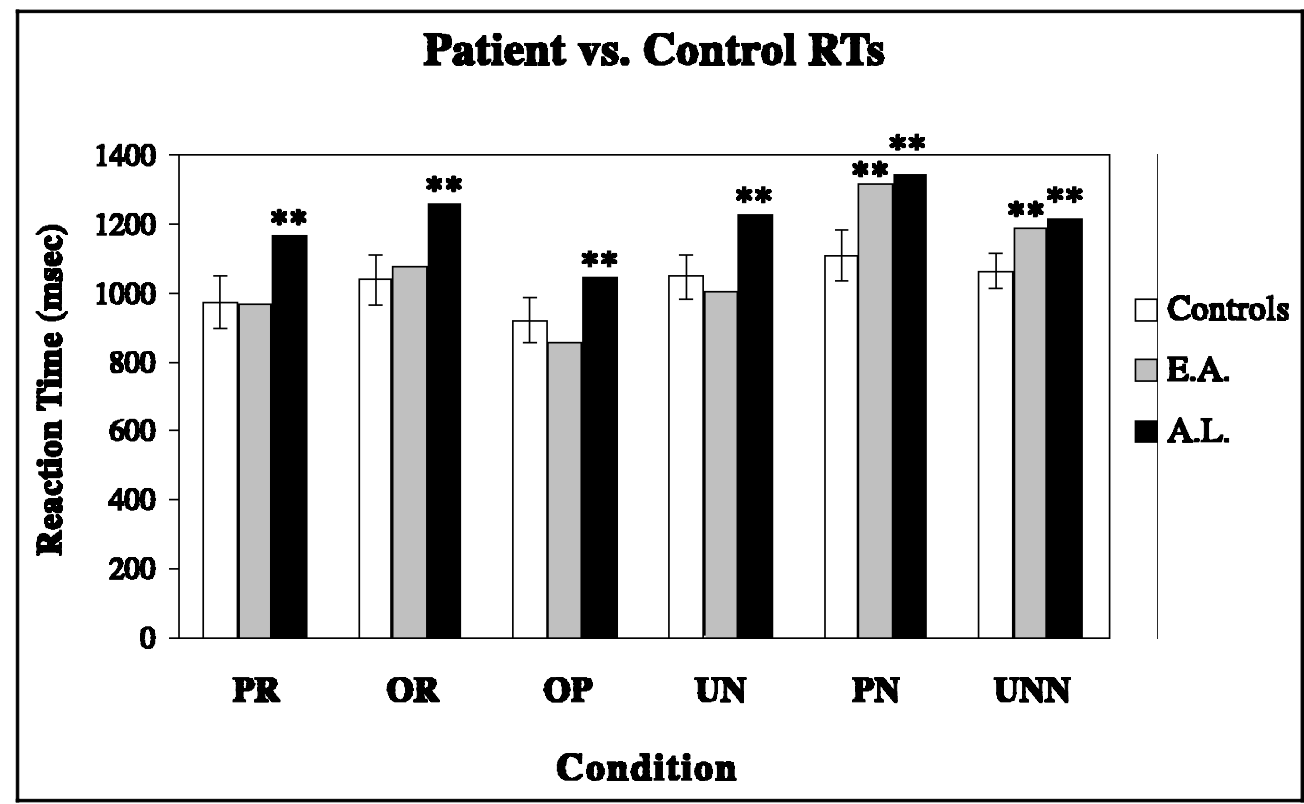




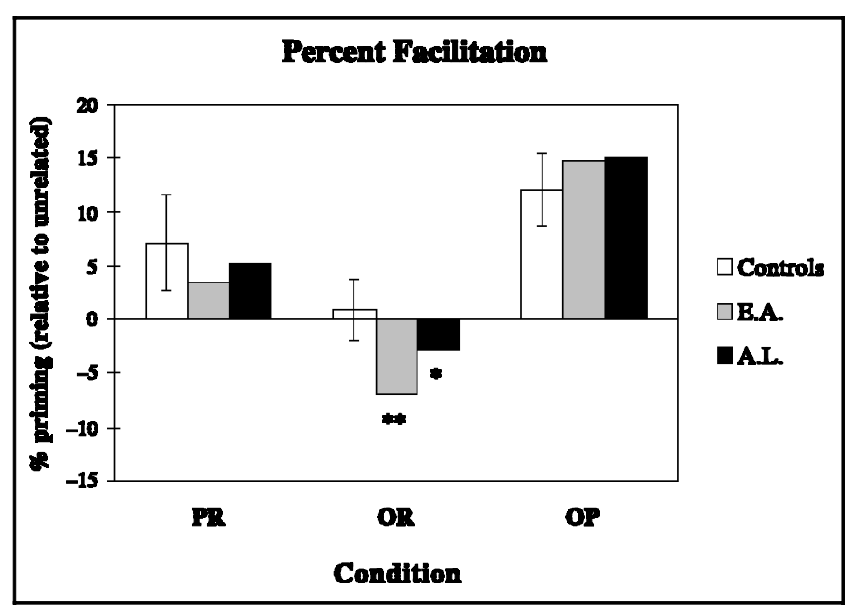

Figure 2. Percent facilitation for controls and patients under each word priming condition, calculated relative to the unrelated word (UN) condition. One asterisk indicates that the patient is outside the $95 \%$ confidence interval, and two asterisks indicate the patient is outside the $99 \%$ confidence interval for controls. The error bars indicate the 95\% confidence interval.

confidence interval (1045 msec) for controls. Because Patient A.L. was slower than controls for all conditions, percent RT facilitation (relative to the UN prime condition) was used as a common metric for comparing RT data across the patients and controls (Figure 2). Repeated-measures ANOVAs with Priming Condition as the within-groups factor and Subject Type as the betweengroups factor were used in computing the statistics that follow. Additionally, we did not collapse across patients when analyzing the data, due to their qualitatively different behavioral presentations. Instead, each patient was compared individually to the control cohort.

For the ANOVA comparing Patient E.A. to controls, there was a main effect of Condition, $F(2,24)=15.35$, $p<.001$, and no Subject Type effect or interaction. For the ANOVA comparing A.L. to controls, there was also a main effect of Condition, $F(2,24)=9.92, p<.006$, and no Subject Type effect or interaction once again. For the $\mathrm{PR}$ and OP prime conditions, both patients were within the 95\% confidence interval of the control subjects. Whereas the OR condition had virtually no effect on controls' RTs (8 msec facilitation), it slowed down the patients' RTs (although not at a significant level for either patient, as revealed by planned comparisons, p's > .14).

We then followed the suggestions of Mycroft, Mitchell, and Kay (2002) for comparing patients and controls in a single ANOVA. According to these authors, provided that the variability of the two groups in question is approximately the same, the differences in group size do not cause a marked departure for the target Type 1 error rate computed using the $F$ criterion. We then calculated the ratio of patient data variance to control data variance in order to determine whether using modified $F$ criteria was needed. For E.A., this ratio was 1.1, which is not problematic for using the standard criterion. For A.L., this ratio was 1.63 , a difference in variance between the two groups in the which the most conservative approach would be to utilize Mycroft et al.'s revised $F$ values. However, the Subject Type analysis was so far from significant, $F(1,24)=.158, p>.70$; there was no need to apply a more stringent $F$ criterion.

\section{Accuracy Data}

The accuracy data are shown in Figure 3. Control accuracy data for real word targets were analyzed using repeated-measures ANOVA. There was no main effect of Prime Type, $F(3,36)=1.81, p>.16$. The average accuracy collapsing across all word priming conditions
Figure 3. Accuracy data for patients and controls for each condition. Overall, the controls were quite well matched to the patients in terms of accuracy. The double pound sign (\#\#) indicates that E.A. actually performed above the $99 \% \mathrm{CI}$ for controls in these conditions.

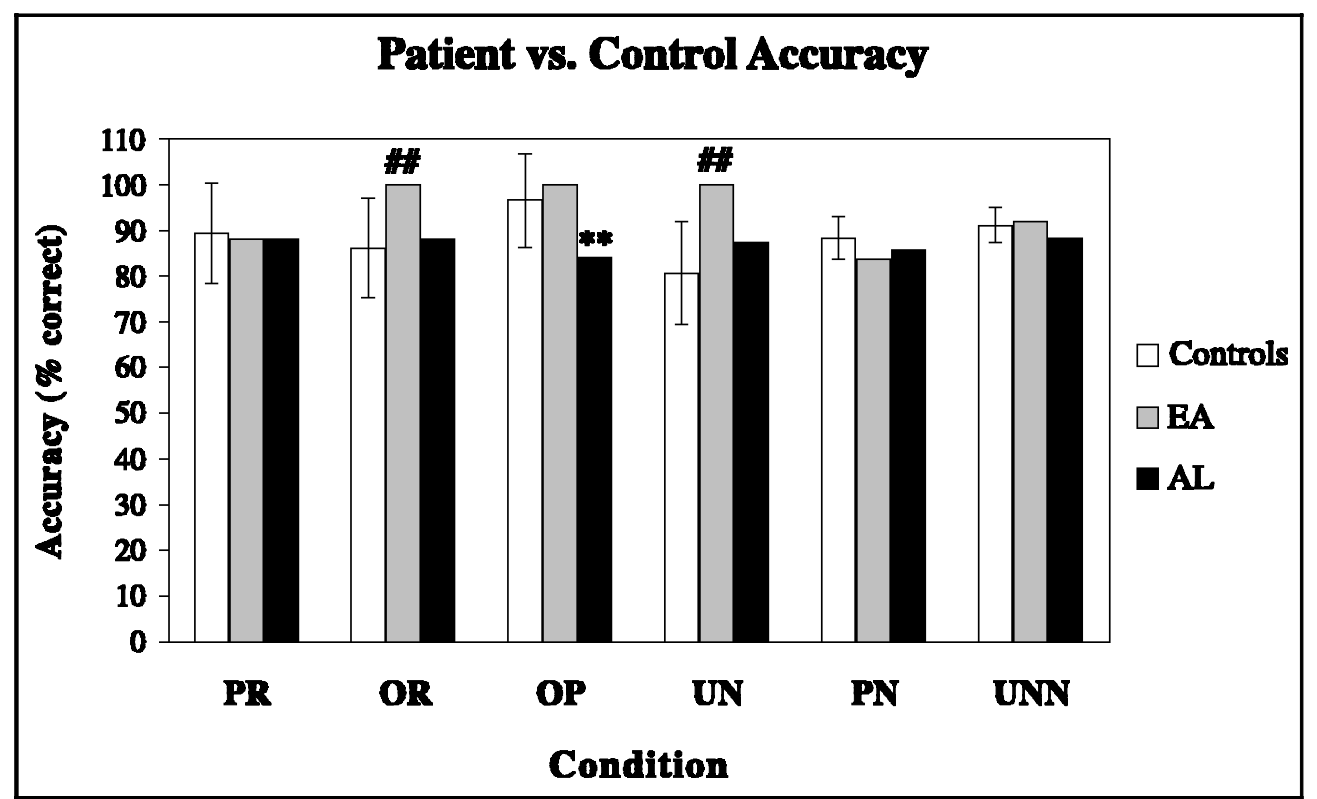


was $88 \%$. Among the alexics, E.A.'s accuracy collapsing across all word priming conditions was $97 \%$, and A.L.'s was $87 \%$. ANOVAs comparing each patient individually to the control cohort revealed no significant effects.

\section{DISCUSSION}

Many studies have explored the role of phonology in the facilitation of lexical decision times (e.g., rhyme priming; Shulman, Hornak, \& Sanders, 1978), but few have examined whether spelling can influence performance for spoken words. We obtained evidence that orthography influenced RTs in an auditory lexical decision task, both in controls and in patients with alexia. Replicating the widely reported rhyme priming effect (Shulman et al., 1978), all participants were quicker to make lexical decisions to words when the prime and the target rhymed. Of particular interest is the role that orthography played in conditions in which the word pairs were OP. Older controls were faster for OP pairs compared to PR pairs, pointing to an additive effect of orthographic and phonological information. This replicates the findings of Baum and Leonard (1999), and lends support to the idea that orthographic information becomes activated as a consequence of auditory lexical access. Donnenwerth-Nolan et al. (1981) suggested that the phonological effects in visual word recognition and the orthographic effects in auditory word recognition are subsumed under a single mechanism that can be explained by Morton's (1969) logogen model. According to this model, the representation of a word in the mental lexicon contains information concerning its spelling, sound, and meaning. When a word is recognized, all three codes become available. This holds regardless of the input modality of the word, since both auditory and visual feature analyzers feed a common set of logogens.

A further issue in the current study concerns the mechanism by which orthographic information entered into the lexical decision-making process. One interpretation is that orthographic and/or phonological priming effects are due to spreading activation in the same way that semantic priming is due to spreading activation along a network in memory organized in terms of semantic relatedness (Tanenhaus et al., 1980). Under this hypothesis, activation of a word might lead to activation of words related along phonological and orthographic networks.

Another possibility is that initial decoding of the prime word starts with some type of feature analysis. Having processed a word with certain orthographic features makes it easier to process a subsequent word with those features; similarly, processing a word with certain phonological features facilitates processing of another word sharing those features (Donnenwerth-Nolan et al., 1981). OP primes benefit from both these dimensions, leading to the fastest response times. Primes that are only PR or only OR can benefit along just one dimension, resulting in less facilitation. The present results showed that PR primes produced significant facilitation, whereas OR primes did not reliably produce any amount of facilitation (or interference) in controls. Thus, phonological relatedness alone was sufficient to facilitate auditory lexical decision, but orthographic relatedness was only influential when combined with phonological relatedness. Clearly, the modality of presentation influences the relative salience of orthography and phonology. In the current experiment, paying attention to the spoken words was necessary to complete the lexical decision task, but accessing the spelling of the words was not. Yet subjects were significantly faster in the OP condition than in the PR condition, suggesting that orthography was activated indirectly by hearing the word.

We now turn to the patient data. This experiment sought to determine whether alexic patients have impaired access to whole-word orthographic information. The alexics' responses under the OP condition were significantly faster than under the PR, OR, and UN conditions, consistent with the performance of controls. The fact that orthographic relatedness influenced response times in both A.L., an LBL reader, and E.A., a global alexic, supports the idea that they can activate orthographic information via auditory input. But what is the nature of these orthographic codes, and what are their neuroanatomical substrates?

Neuroimaging studies have attempted to localize the visual word-form area (VWFA) to a specific brain region, with conflicting results over the last 14 years. The earliest PET studies implicated the left medial extrastriate cortex of the occipital lobe (Petersen, Fox, Posner, Mintun, \& Raichle, 1988; Petersen, Fox, Snyder, \& Raichle, 1990), while subsequent researchers favored the left posterior middle temporal gyrus (Beauregard et al., 1997; Price et al., 1994; Howard et al., 1992). In contrast, support for the classic Dejerine model of reading was obtained by Menard, Kosslyn, Thompson, Alpert, and Rauch (1996), who reported enhanced activity in the left angular gyrus specific for viewing words, compared to viewing pictures, crosshairs, or X's. Another PET study observed that the left medial occipital cortex, as well as the left inferior temporal and fusiform gyri, was activated for both word reading and object naming relative to a nonobject baseline (Bookheimer, Zeffiro, Blaxton, Gaillard, \& Theodore, 1995), leading these authors to doubt the existence of an area specific for the decoding of visual word-forms. Herbster, Mintun, Nebes, and Becker (1997) found activation in the left fusiform gyrus for oral reading of regular and irregular words, but not for pseudowords. They agreed with Bookheimer et al. (1995) that the fusiform activity was related to semantic processing.

Recent fMRI experiments, however, have reported that visually presented words and pseudowords activate an area on or near the left fusiform gyrus in Brodmann's 
areas 37 and/or 19 (Cohen et al., 2000, 2002; Dehaene, Le Clec'H, Poline, Le Bihan, \& Cohen, 2002), suggesting that mid-portion of the left fusiform gyrus may be the VWFA. Polk and Farah (2002) recently demonstrated that this region is not specific for the perceptual form of the stimuli. Words and pseudowords presented in alternating case (i.e., tAbLe) resulted in greater activation than same-case consonant strings (i.e., czrtzy). They argued that this putative word-form area does not respond to the visual form of wordlike stimuli, but rather to a more abstract feature that represents the sequence of abstract letter identities independent of their perceptual features.

This finding is relevant to the current study because both patients have damage to areas 19 and 37. E.A.'s lesion is more extensive and encompasses all but the most lateral portion of this region. Additionally, a neuroanatomical comparison of 10 patients with acquired alexia found that the common lesion cite was the ventral temporal lobe, including the fusiform gyrus (Binder \& Mohr, 1992). Because our patients showed the OP facilitatory effect, it appears they were accessing whole-word orthographic information. It could be that alexics are able to access word orthography via phonology in a top-down fashion, but not able to do so via printed stimuli in a bottom-up fashion. In the alexics, the angular, supramarginal, and superior temporal gyri are intact, thus they have intact phonological processing to allow lexical access from spoken words. Auditory lexical access can result in rapid activation of orthographic information, but the perception of written words does not, since visual information cannot be transmitted in the other direction.

How do the patients' results support, or argue against, some of the major theories that attempt to explain the phenomenon of alexia without agraphia? Warrington and Shallice (1980) have put forth the idea that the visual word-form system is damaged in LBL readers. Our results do not support this hypothesis if the input and output orthographic lexicons are one and the same. Recognizing orthographic similarity between the body of the prime and the target would require an intact wordform system, and the alexics' RTs were influenced by orthographic similarity. Another explanation is that the word-form system itself is intact, but inaccessible via the visual modality due to a disconnection at some stage along the way, whereas the pathway via auditory input is spared. Patterson and Kay (1982) suggested that the visual word-form system itself is intact but disconnected from the letter-form analysis system. This view is parsimonious with the current data, since orthographic access through auditory lexical input does not require identifying letters. Thus, visual orthographic access may be impaired because it requires some sort of feature extraction from individual letters before accessing whole-word orthography, whereas auditory lexical access does not proceed along this same route.
An alternate interpretation is that the OP priming effect does not require on-line access of orthography, but instead is a developmental by-product of learning to read an orthographically inconsistent language. It is possible that the OP rhyme priming benefit from spoken words results from the early importance of onset-rime knowledge in learning to read English (Goswami, 1999). The onset is the initial consonant(s) of a syllable $(c-, b-)$, and the rime is the vowel and any subsequent consonants (-at); rime correspond to rhyme in one-syllable words ("cat," "hat"). Children as young as 3 are aware of these subsyllabic components of speech (Goswami, 1999). The patients were quite literate middle-aged adults when they had their strokes. One could argue that the orthographic effects are not necessarily due to on-line access of orthographic codes, but rather reflect differences in the stability or strength of phonological representations due to orthography (i.e., a long-term memory difference rather than a current access effect). In this scheme, the patients showed the orthography effects because the differences in phonological representations were established prior to their neurological events.

Nevertheless, the present experiment suggests that our alexic patients may have intact orthographic wordform systems, since orthography exerted an influence upon auditory lexical decision times. Additionally, it appears that this system is accessible via auditory input, although it may not be accessible via visual input. This brings us to a critical distinction regarding the nature of orthographic knowledge: Is it best described as a single orthographic lexicon that subserves both reading and spelling (Burt \& Tate, 2002), or as separate input and output lexica (Caramazza, 1988)? This question can also be framed in terms of whether reading and spelling depend on the same processing components or not (Tainturier \& Rapp, 2001). For example, neuropsychological evidence has been used to endorse the view of separate, modality-specific lexical components involved in the recognition (input) and production (output) of written and spoken words (Caramazza, 1988). Others have argued that modality-specific access procedures are used for reading and spelling from a single orthographic processing system (Tainturier \& Rapp, 2001). Because the alexic patients showed an intact OP effect, it could be that (1) feedback access to the unitary orthographic lexicon is available from phonology, but not from visually presented words, or (2) there are separate orthographic lexica for input (reading) and output (writing, spelling). If, in fact, a critical substrate of the visual (or abstract) WFA is localized to the left fusiform gyrus, our results would support the latter idea. In this event, phonological input would activate the orthographic representations used in spelling and writing.

In summary, the data for both controls and alexics support models of word recognition that assume a bidirectional flow of activation between orthography 
and phonology (Ziegler \& Ferrand, 1998; Stone et al., 1997). The present task has shown that the spelling of a word can influence performance on an auditory task; thus, orthography is affecting the perception of spoken words. These results are consistent with developmental perspectives of reading acquisition in children. Initially, the child acquires speech independent of written language, but as the child learns to read and write, a coupling between spoken language and written language forms, and is strengthened as the child's skills improve in both areas (Zecker, 1991). Treiman and Cassar (1997) have suggested that once reading instruction is started, the child ceases to be able to completely dissociate between spelling and sound. This is parsimonious with the idea that over the years, the coupling between letter nodes and phoneme nodes strengthens, such that activation of one necessarily leads to activation of the other. Our findings support Ziegler and Ferrand's (1998) proposal that this mutual activation takes place whether the initial lexical input is presented aurally or visually.

\section{METHODS}

\section{Participants}

Two alexic patients (E.A. and A.L.) with focal lesions of the left medial, inferior temporal-occipital cortex, the posterior hippocampus, and the splenium (Figure 4) participated. In both patients, the damage was the result of a left posterior cerebral artery infarct. The patients tested within normal limits on the Western Aphasia Battery (WAB) and are not classified as aphasics of any type (Table 1), although they exhibited poor performance on the Boston Naming Test (BNT). A group of 13 controls was matched for age (61.8 years), approximate years of education (13.3 years), and handedness. Participants were free from dementia, psychiatric disturbances, and substance abuse. All subjects were paid for their
Figure 4. (A) Images from the MRI scan of E.A., (B) and the CT scan of A.L. These axial sections show the patients' damage in the medial temporaloccipital cortex. E.A.'s lesion is larger than A.L.'s, but both patients sustained damage to the fusiform, lingual, and parahippocampal gyri, the posterior hippocampus, and the splenium of the corpus callosum.

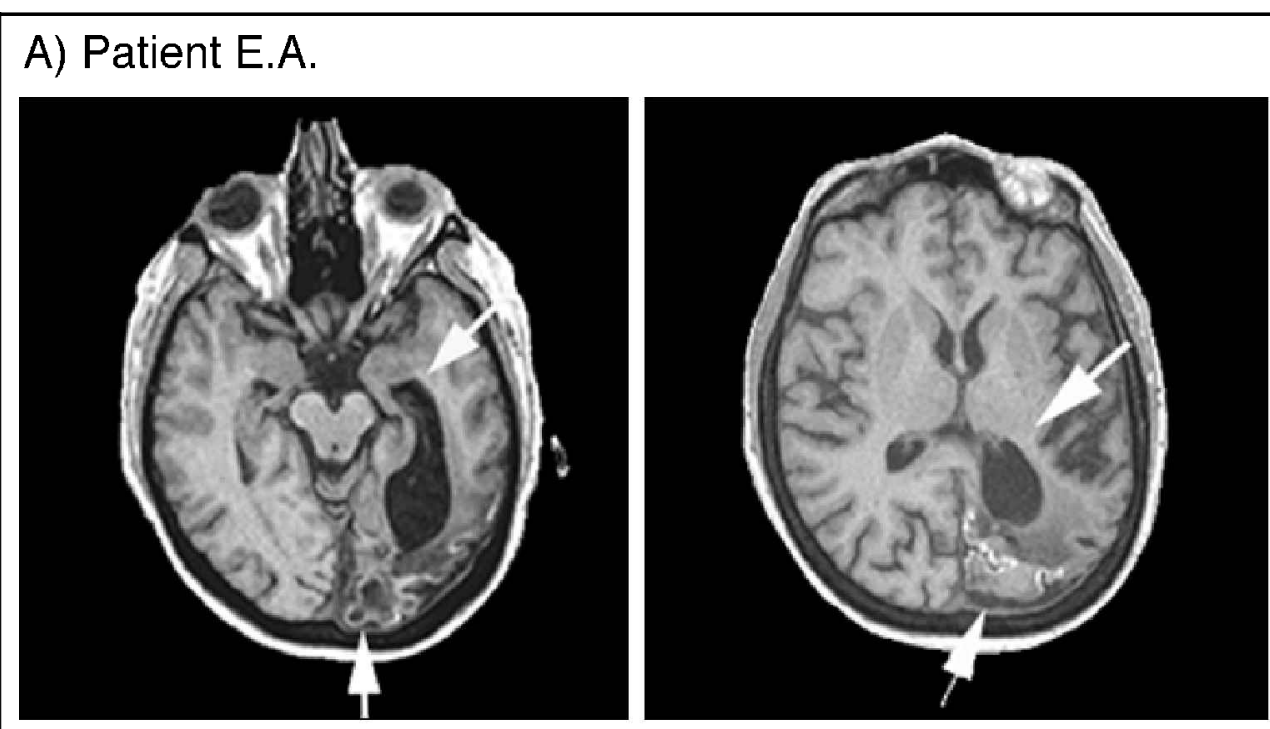

B) Patient A.L.
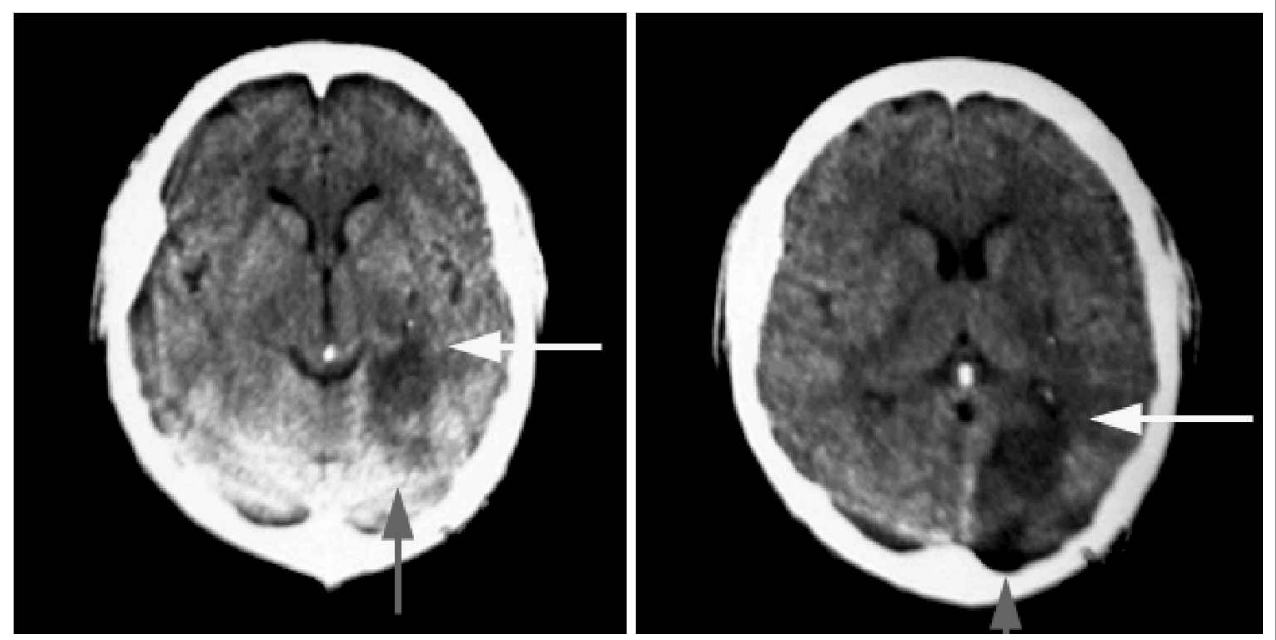
Table 1. Summary Data on Each Patient for Age, Years of Education (Ed), Handedness (Hand), Time Post-onset, and Neuropsychological Testing

\begin{tabular}{|c|c|c|c|c|c|c|c|c|c|c|}
\hline Patient & Age & $E d$ & Hand & Time post-onset (years) & $L M I$ & $L M I I$ & $V R I$ & $V R I I$ & $R C P M$ & $B N T$ \\
\hline E.A. & 54 & 17 & $\mathrm{R}$ & 1.75 & 9 & 9 & 1 & 5 & 50 & $35 / 60\left(1^{\mathrm{st}}\right.$ percentile $)$ \\
\hline A.L. & 69 & 14 & $\mathrm{R}$ & 10.5 & 2 & 1 & 94 & 95 & 90 & $30 / 60\left(1^{\text {st }}\right.$ percentile $)$ \\
\hline Mean & 61.5 & 15.5 & & & & & & & & \\
\hline
\end{tabular}

Percentile scores are given for LM I and LM II (WMS-R Logical Memory I and II); VR I and VR II (WMS-R Visual Reproduction I and II; Patient E.A. was administered WMS-III); and RCPM (Raven's Colored Progressive Matrices). Raw (and percentile) scores are given for BNT.

participation and signed informed consent statements approved by the Institutional Review Boards of the Veterans Affairs Northern California Health Care System and the University of California, Davis.

\section{Patient A.L.}

Patient A.L. is an LBL reader, evidenced by a proportional increase in response latency with the addition of each letter to the word string (e.g., $3678 \mathrm{msec}$ for three-letter words, and $4039 \mathrm{msec}$ for four-letter words). A.L.'s accuracy on single-letter identification (500 msec exposure duration) was $92 \%$. His accuracy on oral reading of common three-letter words (2000 msec exposure) is $75 \%$. In a task in which A.L. was required to name a word spelled aloud to him, he scored 100\% (10/10) accuracy for regular words and $80 \%$ accuracy for irregular words $(8 / 10)$. On a test of oral spelling, he correctly spelled $80 \%$ $(8 / 10)$ of regular and $60 \%(6 / 10)$ of irregular words.

\section{Patient E.A.}

E.A.'s lesion is more extensive than A.L.'s, and her behavioral deficits are larger as well. Her single-letter identification (500 msec exposure duration) was only $20 \%$. We do not report E.A's accuracy on three-letter words because she was unable to read a single word. In contrast, her single-digit number identification at the same exposure time was $80 \%$. Her two-digit and fourdigit number accuracies were $60 \%$ and $2 \%$, respectively. In a test of naming words spelled aloud, E.A. scored $90 \%$ $(9 / 10)$ accuracy for regular words and $80 \%$ accuracy for irregular words (8/10). For oral spelling, she was correct for $80 \%(8 / 10)$ of regular and $70 \%(7 / 10)$ of irregular words. Using the terminology of Binder and Mohr (1992), we refer to E.A. as a global alexic, meaning that she appears to have a deficit at the level of letter naming which precludes her from being able to read in any conscious way. E.A. does demonstrate implicit, or covert, reading, which will be the topic of a separate report (Larsen, Baynes, \& Swick, submitted).

\section{Stimuli and Task Design}

The stimuli consisted of 200 pairs of monosyllabic words or nonwords (100 pairs in which the target was a word, 100 pairs in which the target was a nonword; in all pairs the prime was a word), adapted from Kramer and Donchin (1987) with additions from Dronkers, Redfern, and Ludy (1998), and divided into four blocks of 50 pairs each. Within each of these blocks, the following conditions occurred: (1) target is real word, phonologically related but orthographically dissimilar to prime (PR), e.g., "drawn-gone"; (2) target is real word, orthographically related but phonologically dissimilar to prime (OR), e.g., "hood-food"; (3) target is real word, both phonologically and orthographically related to prime (OP), e.g., "tell-bell"; (4) target is real word, unrelated to prime (UN), e.g., "jazz-globe"; (5) target is nonword, phonologically related to prime (PN), e.g., "sort-bort"; (6) target is nonword, unrelated to prime (UNN), e.g., "filth-gleck." Among the conditions containing a nonword target, there was no manipulation for orthographic relatedness, because there are often multiple ways a nonword may be spelled and there is no agreed-upon "correct" spelling for a nonword. An equal number of trials of each condition took place across blocks.

The stimuli were recorded in a quiet room by an adult male speaker and digitized at a sampling rate of $22 \mathrm{kHz}$ (16 bits, stereo) by an analog-to-digital sound card. The mean length of the prime words was $517 \mathrm{msec}$. The mean durations and frequencies (occurrences/million, Francis \& Kucera, 1982) of the target stimuli were comparable across the six conditions described above, as well as across the word versus nonword categories (Table 2).

Table 2. Mean Stimulus Durations (msec) and Frequencies of Occurrence (Francis \& Kucera, 1982) for Target Stimuli in Each Condition

\begin{tabular}{lcc}
\hline Condition & Duration & Frequency \\
\hline PR & 527.6 & 96.1 \\
OR & 526.3 & 97.8 \\
OP & 529.9 & 95.3 \\
UN & 528.1 & 98.8 \\
PN & 531.1 & \\
UNN & 532.0 & \\
\hline
\end{tabular}




\section{Procedure}

Participants engaged in a lexical decision task in which stimulus pairs were aurally presented over headphones, and they were required to decide if the second word in the pair was a real English word. They indicated their response with a button press on the numberpad of a keyboard using their right hand. They were told to press " 1 " for word, and " 2 " for nonword. Participants were instructed to focus on both accuracy and speed. They were also notified that only their first response would be counted. Each trial started with a brief "ding" sound, indicating that the first prime was about to be presented. The first word of the pair was presented $75 \mathrm{msec}$ after the end of the "ding." The trials were of fixed duration (5900 $\mathrm{msec}$ ), with a stimulus onset asynchrony of $1400 \mathrm{msec}$ between the presentation of the prime and the target. Subtracting the mean duration of the primes, this led to a mean ISI of approximately $883 \mathrm{msec}$. A fixed random order was used in the presentation of the stimuli. A short practice block of 20 stimulus pairs was presented prior to the four test blocks. During the experiment, feedback on performance was given at the end of each block.

\section{Acknowledgments}

This work was supported by Grant DC03424 from the National Institute on Deafness and Communication Disorders and 98-47 CNS-QUA.05 from the James S. McDonnell Foundation. We thank Nina Dronkers and Bob Knight for patient referrals, Rich Ivry for helpful comments, Jary Larsen for clinical testing, and Jonathan Kopelovich for recording the stimuli. Portions of this article were submitted by the first author in satisfaction of the requirements of the Honors Program in Psychology at the University of California, Berkeley.

Reprint requests should be sent to Diane Swick, Department of Neurology (127E), VANCHCS, 150 Muir Road, Martinez, CA 94553, or via e-mail: diane@ebire.org.

\section{REFERENCES}

Baum, S. R., \& Leonard, C. L. (1999). Automatic versus strategic effects of phonology and orthography on auditory lexical access in brain-damaged patients as a function of interstimulus interval. Cortex, 35, 647-660.

Beauregard, M., Chertkow, H., Bub, D., Murtha, S., Dixon, R., \& Evans, A. (1997). The neural substrate for concrete, abstract, and emotional word lexica: A positron emission tomography study. Journal of Cognitive Neuroscience, 9, 441-461.

Behrmann, M., Plaut, D. C., \& Nelson, J. (1998). A review and new data supporting an interactive account of letter-by-letter reading. Cognitive Neuropsychology, 15, 7-51.

Binder, J. R., \& Mohr, J. P. (1992). The topography of callosal reading pathways. Brain, 115, 1807-1826.

Bookheimer, S. Y., Zeffiro, T. A., Blaxton, T., Gaillard, W., \& Theodore, W. (1995). Regional cerebral blood flow during object naming and word reading. Human Brain Mapping, 3, 93-106.

Burt, J. S., \& Tate, H. (2002). Does a reading lexicon provide orthographic representations for spelling? Journal of Memory and Language, 46, 518-543.
Caramazza, A. (1988). Some aspects of language processing revealed through the analysis of acquired aphasia: The lexical system. Annual Review of Neuroscience, 11, 395-421.

Carr, T. H., \& Pollatsek, A. (1985). Recognizing printed words: A look at current models. In D. Besner, T. G. Waller, \& G. E. MacKinnon (Eds.), Reading research: Advances in theory and practice 5 (pp. 1-82). San Diego: Academic Press.

Clark, H. H. (1973). The language-as-fixed-effect fallacy: A critique of language statistics in psychological research. Journal of Verbal Learning and Verbal Behavior, 12, 335-359.

Cohen, L., Dehaene, S., Naccache, L., Lehéricy, S., Dehaene-Lambertz, G., Henaff, M. A., \& Michel, F. (2000). The visual word form area: Spatial and temporal characterization of an initial stage of reading in normal subjects and posterior split-brain patients. Brain, 123, 291-307.

Cohen, L., Lehéricy, S., Chochon, F., Lemer, C., Rivaud, S., \& Dehaene, S. (2002). Language-specific tuning of visual cortex? Functional properties of the visual word form area. Brain, 125, 1054-1069.

Coltheart, M., Curtis, B., Atkins, P., \& Haller, M. (1991). Models of reading aloud: Dual-route and parallel-distributed processing approaches. Psychological Review, 100, 589-608.

Dehaene, S., Le Clec'H, G., Poline, J.B., Le Bihan, D., \& Cohen, L. (2002). The visual word form area: A prelexical representation of visual words in the fusiform gyrus. NeuroReport, 13, 321-325.

Dejerine, J. (1892). Contribution a l'etude anatomopathologique et clinique des differentes varietes de cecite verbale. Comptes Rendus Hebdomadaires des Seances et Memoires de la Societe de Biologie, 4, 61-90.

Donnenwerth-Nolan, S., Tanenhaus, M. K., \& Seidenberg, M. S. (1981). Multiple code activation in word recognition: Evidence from rhyme monitoring. Journal of Experimental Psychology: Human Learning and Memory, 7, 170-180.

Dronkers, N. F., Redfern, B. B., \& Ludy, C. (1998). Brain regions associated with conduction aphasia and echoic rehearsal. Journal of the International Neuropsychological Society, 4 , 23-24.

Francis, W. N., \& Kucera, H. (1982). Frequency analysis of English usage: Lexicon and grammar. Boston: Houghton Mifflin.

Goswami, U. (1999). Integrating orthographic and phonological knowledge as reading develops: Onsets, rimes and analogies in children's reading. In R. M. Klein \& P. A. McMullen (Eds.), Converging methods for understanding reading and dyslexia (pp. 57-75). Cambridge: MIT Press.

Herbster, A. N., Mintun, M. A., Nebes, R. D., \& Becker, J. T. (1997). Regional cerebral blood flow during word and nonword reading. Human Brain Mapping, 5, 84-92.

Howard, D., Patterson, K., Wise, R., Brown, W. D., Friston, K. Weiller, C., \& Frackowiak, R. (1992). The cortical localization of the lexicons: Positron emission tomography evidence. Brain, 115, 1769-1782.

Jacobs, A. M., \& Grainger, J. (1994). Models of word recognition-sampling the state of the art. Journal of Experimental Psychology: Human Perception and Performance, 20, 1311-1334.

Jakimik, J., Cole, R., \& Rudnicky, A. (1985). Sound and spelling in spoken word recognition. Journal of Memory and Language, 24, 165-178.

Kramer, A. F., \& Donchin, E. (1987). Brain potentials as indices of orthographic and phonological interaction during word matching. Journal of Experimental Psychology: Learning, Memory, and Cognition, 13, 76-86. 
Larsen, J., Baynes, K., \& Swick, D. (submitted). Right hemisphere reading mechanisms in a global alexic patient.

Leonard, C. L., \& Baum, S. R. (1997). The influence of phonological and orthographic information on auditory lexical access in brain-damaged patients: A preliminary investigation. Aphasiology, 11, 1031-1041.

Menard, M. T., Kosslyn, S., Thompson, W. L., Alpert, N. M., \& Rauch, S. L. (1996). Encoding words and pictures: A positron emission tomography study. Neuropsychologia, 34, 185-194.

Miozzo, M., \& Caramazza, A. (1998). Varieties of pure alexia: The case of failure to access graphemic representations. Cognitive Neuropsychology, 15, 203-238.

Morton, J. (1969). Interaction of information in word recognition. Psychological Review, 76, 165-178.

Mycroft, R. H., Mitchell, D. C., \& Kay, J. (2002). An evaluation of statistical procedures for comparing an individual's performance with that of controls. Neuropsychologia, 19, 291-299.

Norris, D., McQueen, J. M., \& Cutler, A. (2000). Merging information in speech recognition: Feedback is never necessary. Behavioral and Brain Sciences, 23, 299-370.

Patterson, K., \& Kay, J. (1982). Letter-by-letter reading: Psychological descriptions of a neurological syndrome. Quarterly Journal of Experimental Psychology, 34A, 411-441.

Petersen, S. E., Fox, P. T., Posner, M. I., Mintun, M., \& Raichle, M. E. (1988). Positron emission tomographic studies of the cortical anatomy of single-word processing. Nature, 331, 585-589.

Petersen, S. E., Fox, P. T., Snyder, A. Z., \& Raichle, M. E. (1990). Activation of extrastriate and frontal cortical areas by visual words and word-like stimuli. Science, 249, 1041-1044.

Plaut, D. C., McClelland, J. L., Seidenberg, M. S., \& Patterson, K. (1996). Understanding normal and impaired word reading: Computational principles in quasi-regular domains. Psychological Review, 103, 56-115.

Polk, T. A., \& Farah, M. J. (2002). Functional MRI evidence for an abstract, not perceptual, word-form area. Journal of Experimental Psychology: General, 131, 65-72.
Price, C. J., Wise, R. J. S., Watson, J. D. G., Patterson, K., Howard, D., \& Frackowiak, R. S. J. (1994). Brain activity during reading: The effects of exposure duration and task. Brain, 117, 1255-1269.

Saffran, E. M., \& Coslett, H. B. (1998). Reading in pure alexia. Cognitive Neuropsychology, 15, 141-165.

Seidenberg, M. S., \& McClelland, J. L. (1989). A distributed, developmental model of word recognition and naming. Psychological Review, 96, 523-568.

Seidenberg, M. S., \& Tanenhaus, M. K. (1979). Orthographic effects on rhyme monitoring. Journal of Experimental Psychology: Human Learning and Memory, 5, 546-554.

Shulman, H., Hornak, R., \& Sanders, M. (1978). The effects of graphemic, phonetic, and semantic relationships on access to lexical structures. Memory and Cognition, 6, $115-123$.

Stone, G. O., Vanhoy, M., \& Van Orden, G. C. (1997). Perception is a two-way street: Feedforward and feedback phonology in visual word recognition. Journal of Memory and Language, 36, 337-359.

Tainturier, M.-J., \& Rapp, B. (2001). The spelling process. In B. Rapp (Ed.), The handbook of cognitive neuropsychology: What deficits reveal about the human mind (pp. 263-289). Philadelphia: Psychology Press/Taylor \& Francis.

Tanenhaus, M. K., Flanigan, H. P., \& Seidenberg, M. S. (1980). Orthographic and phonological activation in auditory and visual word recognition. Memory and Cognition, 8, 513-520.

Treiman, R., \& Cassar, M. (1997). Can children and adults focus on sounds as opposed to spelling in a phoneme counting task? Developmental Psychology, 33, 771-780.

Warrington, E. K., \& Shallice, T. (1980). Word-form dyslexia. Brain, 103, 99-112.

Zecker, S. G. (1991). The orthographic code: Developmental trends in reading-disabled and normally-achieving children. Annals of Dyslexia, 41, 178-192.

Ziegler, J. C., \& Ferrand, L. (1998). Orthography shapes the perception of speech: The consistency effect in auditory word recognition. Psychonomic Bulletin and Review, 5, 683-689. 